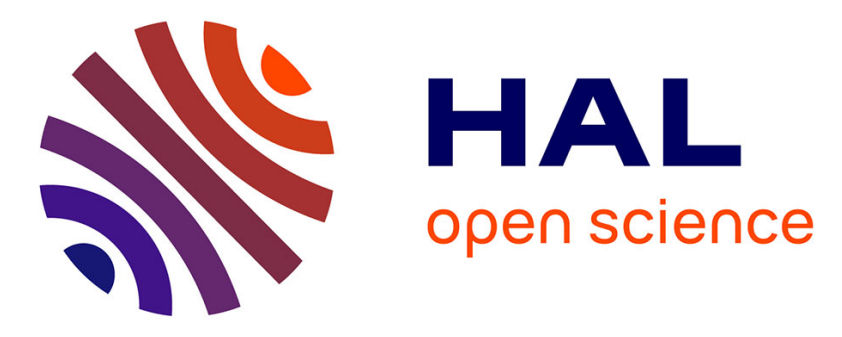

\title{
Youla-Kucera control structures for switching
}

Francisco Navas, Imane Mahtout, Vicente Milanés, Fawzi Nashashibi

\section{To cite this version:}

Francisco Navas, Imane Mahtout, Vicente Milanés, Fawzi Nashashibi. Youla-Kucera control structures for switching. CCTA 2018 - 2nd IEEE Conference on Control Technology and Applications, Aug 2018, Copenhagen, Denmark. hal-01801224

\section{HAL Id: hal-01801224 \\ https://hal.inria.fr/hal-01801224}

Submitted on 28 May 2018

HAL is a multi-disciplinary open access archive for the deposit and dissemination of scientific research documents, whether they are published or not. The documents may come from teaching and research institutions in France or abroad, or from public or private research centers.
L'archive ouverte pluridisciplinaire HAL, est destinée au dépôt et à la diffusion de documents scientifiques de niveau recherche, publiés ou non, émanant des établissements d'enseignement et de recherche français ou étrangers, des laboratoires publics ou privés. 


\title{
Youla-Kucera control structures for switching
}

\author{
Francisco Navas ${ }^{1}$, Imane Mahtout ${ }^{2}$, Vicente Milanés ${ }^{2}$ and Fawzi Nashashibi ${ }^{1}$
}

\begin{abstract}
This paper explores the different controller structures in connection with Youla-Kucera (YK) parameterization to provide stable controller reconfiguration. Mathematical stability proof is given for each of the structures. Even if stability is guaranteed, transient effects of switching between controllers can become important for the system performance. Thus, transient behavior depending on the control structure is also analysed. A comparative study is carried out among all the structures, so guidelines are given on how to choose the appropriate YK control structure.
\end{abstract}

\section{INTRODUCTION}

Computer-controlled systems (i.e. humanoids, automated vehicles) are getting more and more complex, demanding advanced control algorithms able to reach the performance goals. This computer-controlled system are alive, meaning that the plant can change in time, potential faults must be properly handled or even new sensor/actuators can be added [1]. Such systems require a control technique able to perform controller reconfiguration depending on these events.

Controller reconfiguration goes from switching between two fixed linear controllers to interpolation of control signals generated by different single controllers. However, stability is a commom problem in controller reconfiguration applications. A linear combination of two stabilizing controllers could not result in a stabilizing controller [2]. 40 years ago, the study of coprime factorizations of linear plants led to a theory giving the class of all stabilizing controllers for a linear plant: Youla-Jabr-Bongiorno-Kucera (YK) parameterization [3]. Stable controller switching is possible by using the class of all stabilizing controllers, as first mentioned in [4]. Stability proof can be found in [5]; later extended under arbitrary change of the switching signal over time in [6].

YK has solved problems in different control areas: High performance control blends strenghs of classical, optimal and robust control through YK theory. The idea is to obtain high performance on the face of uncertainty and change in a plant [7]; a fault tolerant control structure with a direct separation in terms of fault signature, useful to fault detection and consequent controller reconfiguration [8]; or Plug\&Play control [9], which introduces new sensors/actuators, providing stabilizing guarantees during the transition phase as well as retaining the original control structure.

${ }^{1}$ F. Navas and F. Nashashibi are with the Robotics and Intelligent Transportation Systems (RITS) team, Inria Paris, 56 rue du Charolais, 75012 Paris, France francisco.navas-matos, fawzi.nashashibi Cinria.fr

${ }^{2}$ I. Mahtout and V. Milanés are with the Research Department, Renault SAS, 1 Avenue de Golf, 78280 Guyancourt, France imane. mahtout, vicente.milanes @renault.com
But some drawbacks come up when using the YK architecture: complexity of resulting controller, matrix inversability or disconnection of the plant for implementation. The standard structure in [4] has been modified in the literature to avoid matrix inversability [10], plant disconnection [11] [12], or to reduce the complexity of the resulting controller [13].

The main focus in this paper is to summarize all these structures, highlighting the solved problems, and providing mathematical proof that stability remains. A partial comparison between some structures has been already adressed in [13], but it does not include all the structures in the literature, stability proof is not given, and the transient behavior is not analysed. Even if transitions are stable, transient effects of switching between controllers can become important for the system performance. Thus, transient responses are studied for all the YK control structures, analyzing their performance.

The paper is organized as follows: Section II introduces the YK mathematical basis. Control structures in this section correspond to those that appeared for the first time in [4]. Modifications of these structures to avoid some of the associated problems to YK are shown in section III. Section IV compares the transient response of all the structures through a numerical example. To finish, concluding remarks are given in section $\mathrm{V}$.

\section{YOULA-KUCERA CONTROLLER MODIFICATION}

This section introduces the mathematical basis in which Youla-Kucera (YK) relies on for making stable transition between different controllers. The change between both controllers is made through the parameterization of all the stabilizing controllers for a given plant. This parameterization is based on the doubly coprime factorization [14].

The notation is standard; $\mathbb{R} H_{\infty}$ is the real stable transfer function space; $G$ represents the input/output behavior or internal dynamics of the physical system to control. In the case of LTI continous systems, the state space representation of $G$ yields:

$$
\begin{aligned}
& \dot{x}(t)=A x(t)+B u(t) \\
& y(t)=C x(t)+D u(t)
\end{aligned}, \quad G(s)=\left[\begin{array}{l|l}
A & B \\
\hline C & D
\end{array}\right]
$$

where $t$ indicates time, $x(t)$ is the state vector, $\dot{x}(t)=d x / d t$ is the evolution over time of the state vector, $y(t)$ the measurement vector and $u(t)$ the control vector. Coefficients $A, B, C$ and $D$ are constant matrices with the corresponding dimensions. $n$ is the plant order.

The plant could be stabilized by any appropriate LTI 
controller $K_{i}$ in state-space:

$$
\begin{aligned}
\dot{x}(t) & =A_{i}^{c} x(t)+B_{i}^{c} y(t) \\
u(t) & =C_{i}^{c} x(t)+D_{i}^{c} y(t)
\end{aligned}, \quad K_{i}(s)=\left[\begin{array}{c|c}
A_{i}^{c} & B_{i}^{c} \\
\hline C_{i}^{c} & D_{i}^{c}
\end{array}\right]
$$

where $A_{i}^{c}, B_{i}^{c}, C_{i}^{c}$ and $D_{i}^{c}$ are constant matrices for each controller $K_{i} . m_{i}$ denotes controller order.

Assuming that $K_{0}$ corresponds to an initial controller and $K_{1}$ to a final one, it is possible to switch between them in a stable way thanks to the $\mathrm{YK}$ parameterization of all controllers.

The plant $G$ and both controllers $K_{0}$ and $K_{1}$ must be factorized to apply the Youla-Kucera theory.

\section{A. Doubly coprime factorization}

For single-input single-output (SISO) systems, factorization leads to plant and controller being represented as the product of two transfer functions. Coprimeness refers to the absence of common zeros in the right half-plane.

For multi-input multi-output (MIMO) systems, factorization is represented as the ratio between a stable matrix transfer function with a matrix transfer function inversely stable. Coprimeness is expressed as a full rank condition on the matrices outside of the unit circle.

In both cases, double coprimeness excludes unstable pole/zero cancellations, and refers to the idea of being right and left coprime [15]. Definitions and theorem below are applicable for both SISO and MIMO systems:

Definition 1: Two different matrices $M_{i}$ and $N_{i}$ are right coprimes over $\mathbb{R} H_{\infty}^{\text {pxm }}$ if they have the same number of columns and if matrices $X_{r, i}$ and $Y_{r, i}$ exist such that:

$$
\left[\begin{array}{ll}
X_{r, i} & Y_{r, i}
\end{array}\right]\left[\begin{array}{c}
M_{i} \\
N_{i}
\end{array}\right]=X_{r, i} M_{i}+Y_{r, i} N_{i}=I
$$

Definition 2: Two different matrices $\tilde{M}_{i}$ and $\tilde{N}_{i}$ are left coprimes over $\mathbb{R} H_{\infty}^{p x m}$ if they have the same number of rows and if matrices $X_{l, i}$ and $Y_{l, i}$ exist such that:

$$
\left[\begin{array}{ll}
\tilde{M}_{i} & \tilde{N}_{i}
\end{array}\right]\left[\begin{array}{c}
X_{l, i} \\
Y_{l, i}
\end{array}\right]=\tilde{M}_{i} X_{l, i}+\tilde{N}_{i} Y_{l, i}=I
$$

These coprime factors should be such that $G, K_{0}$ and $K_{1}$ are:

$$
\begin{array}{r}
G=N_{i} M_{i}^{-1}=\tilde{M}_{i}^{-1} \tilde{N}_{i} \\
K_{i}=U_{i} V_{i}^{-1}=\tilde{V}_{i}^{-1} \tilde{U}_{i} \\
\text { with } \quad i=0,1
\end{array}
$$

At the same time, these coprime factors $U_{i}, \tilde{U}_{i}, V_{i}, \tilde{V}_{i}$, $N_{i}, \tilde{N}_{i}, M_{i}$ and $\tilde{M}_{i} \in \mathbb{R} H_{\infty}$ must satisfy the double Bézout's identity [16]:

$\left[\begin{array}{cc}\tilde{V}_{i} & -\tilde{U}_{i} \\ -\tilde{N}_{i} & \tilde{M}_{i}\end{array}\right]\left[\begin{array}{cc}M_{i} & U_{i} \\ N_{i} & V_{i}\end{array}\right]=\left[\begin{array}{cc}M_{i} & U_{i} \\ N_{i} & V_{i}\end{array}\right]\left[\begin{array}{cc}\tilde{V}_{i} & -\tilde{U}_{i} \\ -\tilde{N}_{i} & \tilde{M}_{i}\end{array}\right]=\left[\begin{array}{cc}I & 0 \\ 0 & I\end{array}\right]$

Theorem 1 permits to obtain coprime factors for $\left[G, K_{0}\right]$ and $\left[G, K_{1}\right]$, when $G, K_{0}$ and $K_{1}$ are described in state-space form. These factors satisfy (5) and (6) (further details can be found in [7]):
Theorem 1: Consider a plant in state space representation as $G=C(z I-A)^{-1} B+D$ with $A, B, C$ stabilizable and detectable, and a stabilizing controller $K_{i}=C_{i}^{c}(z I-$ $\left.A_{i}^{c}\right)^{-1} B_{i}^{c}+D_{i}^{c} . F_{i}$ and $F_{i}^{c}$ should be chosen such that $A+B F_{i}$ and $A_{i}^{c}+B_{i}^{c} F_{i}^{c} \in \mathbb{R} H_{\infty}^{p x m}$. Coprime factors are given by:

$$
\left[\begin{array}{cc}
M_{i} & U_{i} \\
N_{i} & V_{i}
\end{array}\right]=\left[\begin{array}{cc|cc}
A+B F_{i} & 0 & B & 0 \\
0 & A_{i}^{c}+B_{i}^{c} F_{i}^{c} & 0 & B_{i}^{c} \\
\hline F_{i} & C_{i}^{c}+D_{i}^{c} F_{i}^{c} & I & D_{i}^{c} \\
C+D F_{i} & F_{i}^{c} & D & I
\end{array}\right]
$$

$$
\begin{aligned}
& {\left[\begin{array}{cc}
\tilde{V}_{i} & -\tilde{U}_{i} \\
-\tilde{N}_{i} & \tilde{M}_{i}
\end{array}\right]=} \\
& {\left[\begin{array}{cc|cc}
A+B Y_{i} D_{i}^{c} C & B Y_{i} C_{i}^{c} & -B Y_{i} & B Y_{i} D_{i}^{c} \\
B_{i}^{c} Z_{i} C & A_{i}^{c}+B_{i}^{c} Z_{i} D C_{i}^{c} & -B_{i}^{c} Z_{i} D & B_{i}^{c} Z_{i} \\
\hline F_{i}-Y_{i} D_{i}^{c} C & -C_{i}^{c} & I & -D_{i}^{c} \\
C & -F_{i}^{c} & 0 & I
\end{array}\right]} \\
& \text { with } Y_{i}=\left(I-D_{i}^{c} D\right)^{-1} \quad \text { and } \quad Z_{i}=\left(I-D D_{i}^{c}\right)^{-1}
\end{aligned}
$$

An non-minimal realization for the doubly coprime factors shown in Eqs. (7) and (8) results in an order equivalent to $n+m_{i}$.

\section{B. Youla-Kucera parameterization}

YK parameterization provides all stabilizing controllers for a given plant $G$, by interconnecting an initial controller $K_{0}$ with a parameter $Q$, called YK parameter, which can be any stable system with appropriate dimensions.

Theorem 2: Consider a fixed plant $G$ connected to an initial controller $K_{0}$ described by their coprime factors $G=$ $N_{0} M_{0}^{-1}=\tilde{M}_{0}{ }^{-1} \tilde{N}_{0}$ and $K_{0}=U_{0} V_{0}^{-1}=\tilde{V}_{0}{ }^{-1} \tilde{U}_{0}$. Then the set of all stabilizing controllers for $G$ is described by:

$$
\begin{gathered}
K(Q)=\left(U_{0}+M_{0} \gamma Q\right)\left(V_{0}+N_{0} \gamma Q\right)^{-1}= \\
=\left(\tilde{V}_{0}+\gamma Q \tilde{N}_{0}\right)^{-1}\left(\tilde{U}_{0}+\gamma Q \tilde{M}_{0}\right), \quad Q \in \mathbb{R} H_{\infty}
\end{gathered}
$$

From this general description is possible to change from an initial controller $K_{0}$ to $K_{1}$ online, without losing stability by choosing the appropriate $Q$. It should be calculated as follows [2]:

Theorem 3: Let $G=N_{0} M_{0}^{-1}=\tilde{M}_{0}^{-1} \tilde{N}_{0}$ be a coprime factorization of the plant $G$ and $K_{0}=U_{0} V_{0}^{-1}=\tilde{V}_{0}{ }^{-1} \tilde{U}_{0}$ an initial stabilizing controller. A second controller is given by $K_{1}=U_{1} V_{1}^{-1}=\tilde{V}_{1}^{-1} \tilde{U}_{1} . Q$ is calculated as:

$$
Q=X_{1}\left(\tilde{U}_{1} V_{0}-\tilde{V}_{1} U_{0}\right)
$$

with $X_{1}=M_{0}^{-1} M_{1}$.

As coprime factors are stable, the transfer function $X_{1}$ is stable. In some special cases, coprime factors will coincide $M_{0}=M_{1}$ and therefore $X_{1}=I$.

From these two theorems, the standard control structures for controller switching are derived. Figure 1 shows the control structure for switching based on right coprime factors. On the other hand, the structure in fig. 2 depends on left coprime factors. As standard, one means the very first YK control structures that appeared in [4]. The complexity of these control structure is defined as the order/state dimension of the switched controller $K(Q)$ : A non-minimal realization 


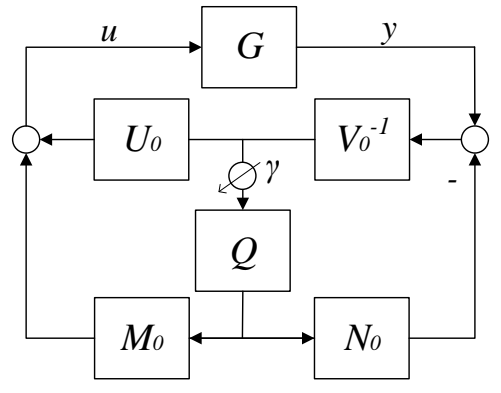

Fig. 1. Structure 1.

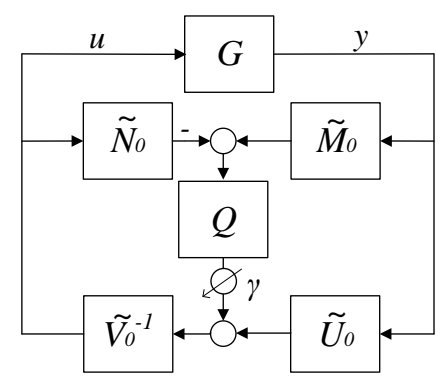

Fig. 2. Structure 2.

of structure 1 and 2 may yield a $K(Q)$ with $7\left(n+m_{0}\right)+$ $3\left(n+m_{1}\right)$ states.

When doing controller transitions, the scalar factor $\gamma$ plays a key role. It regulates the different level of activation of the YK parameter $Q$. $\gamma$ may vary from 0 to 1 , being 0 a $100 \%$ contribution of $K_{0}$ and 1 a $100 \%$ contribution of $K_{1}$. In short, $\gamma$ is the switching signal between $K_{0}$ and $K_{1}$.

The stability in the transition is ensured when $Q \in \mathbb{R} H_{\infty}$, so any linear combination of $Q$ and $\gamma$ will provide stable responses. Independently of the value of $\gamma$, the closed-loop (CL) poles during the transition will be the combination of the CL poles of $\left[G, K_{0}\right]$ and $\left[G, K_{1}\right]$. Proof relies on the controller description $K(Q)$ in eq. (9). Further details can be found in [5].

\section{MODIFIED CONTROLLER ARCHITECTURES FOR SWITCHING}

Fig. 1 represents the YK implementation for controller reconfiguration based on right coprime factors, whereas Fig. 2 depicts the YK control structure for switching based on left coprime factors. Both implementations present some drawbacks such a high order complexity of $K(Q)$, matrices' inversability or controller design from scratch. These structures are modified in [12] [10] [13], so the associated problems can be suppressed. These are explained below, highlighting the solved problems and providing mathematical proof that transition between controllers is stable.

\section{A. Structures 3 and 4}

Controller reconfiguration using structures 1 and 2 requires the initial controller $K_{0}$ to be divided in its coprime factors $U_{0}, V_{0}$ or $\tilde{U}_{0}$ and $\tilde{V}_{0}$. Even if the system is already operational with an initial controller $K_{0}$, this one should be disconnected.

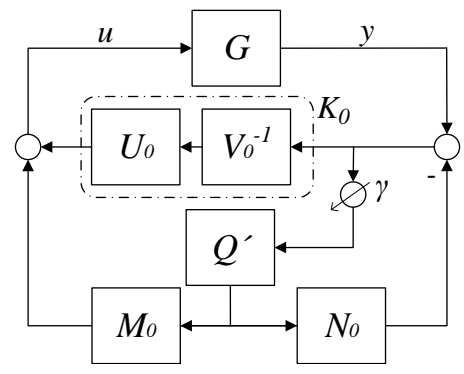

Fig. 3. Structure 3.

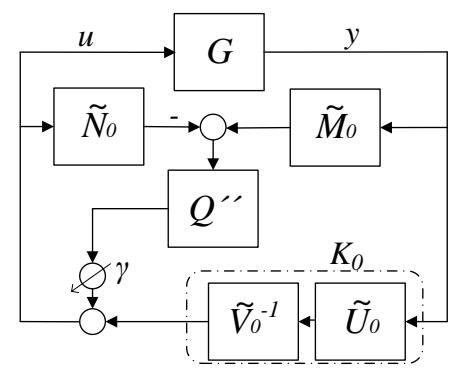

Fig. 4. Structure 4.

This is unfeasible if the system shutdown is very expensive, or the initial controller is part of a safety circuit.

[12] keeps the initial controller in place, accessing at its terminal to carry out the YK controller reconfiguration. New control dynamics can be added online, without removing the original controller. This allows to return to the original controller in case of problems with the new one. Control structures for right and left coprime factorizations are shown in Figs. 3 and 4 respectively. As $K_{0}$ is not decomposed in coprime factors, inversion of matrices is no longer needed; and the complexity of the resulting $K(Q)$ is lower: $5(n+$ $\left.m_{0}\right)+3\left(n+m_{1}\right)+m_{0}$. Please notice that the calculation of the YK parameter $Q$ differs from the standard one in eq. (10):

Theorem 4: Let $G=N_{0} M_{0}^{-1}=\tilde{M}_{0}^{-1} \tilde{N}_{0}$ be a coprime factorization of the plant $G$ and $K_{0}=U_{0} V_{0}^{-1}=\tilde{V}_{0}^{-1} \tilde{U}_{0}$ an initial stabilizing controller that can not be disconnected. A second controller is given by $K_{1}=U_{1} V_{1}^{-1}=\tilde{V}_{1}^{-1} \tilde{U}_{1}$.

When using right coprime factors $M_{0}$ and $N_{0}, Q^{\prime}$ is calculated as:

$$
Q^{\prime}=Q V_{0}^{-1}=X_{1}\left(\tilde{U}_{1}-\tilde{V}_{1} \tilde{U}_{0} \tilde{V}_{0}^{-1}\right)
$$

When using left coprime factors $\tilde{M}_{0}$ and $\tilde{N}_{0}, Q^{\prime \prime}$ is calculated as:

$$
Q^{\prime \prime}=Q \tilde{V}_{0}^{-1}=X_{1}\left(\tilde{V}_{0}^{-1}\left(\tilde{U}_{1} V_{0}-\tilde{V}_{1} U_{0}\right)\right)
$$

with $X_{1}=M_{0}^{-1} M_{1}$.

Once structures 3 and 4 are defined, the YK property of stable controller reconfiguration is verified. Stability has already been demonstrated in the literature (see [5]) for structures 1 and 2 through eq. (9). To prove that stability is still preserved in these new structures, it is only necessary to check that $K(Q)$ remains as in eq. (9). 


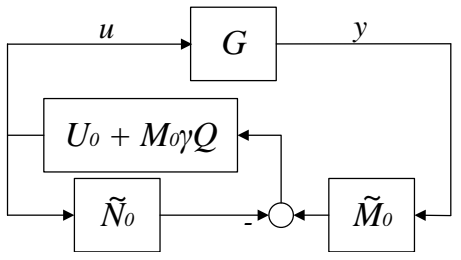

Fig. 5. Structure 5 .

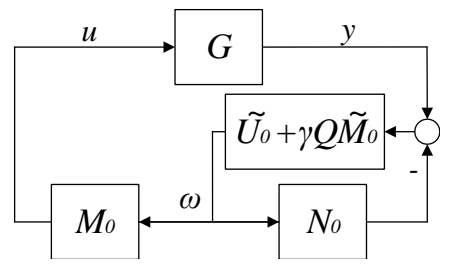

Fig. 6. Structure 6 .

According to the block diagram of structure 3 (fig. 3), $K(Q)$ yields:

$$
\begin{array}{r}
u=\left(K_{0}+M_{0} \gamma Q^{\prime}\right)\left(I+N_{0} \gamma Q^{\prime}\right)^{-1} y \\
u=\left(U_{0} V_{0}^{-1}+M_{0} \gamma Q^{\prime}\right)\left(I+N_{0} \gamma Q^{\prime}\right)^{-1} y \\
u=\left(U_{0} V_{0}^{-1}+M_{0} \gamma Q V_{0}^{-1}\right)\left(I+N_{0} \gamma Q V_{0}^{-1}\right)^{-1} y \\
u=\left(U_{0}+M_{0} \gamma Q\right)\left(V_{0}+N_{0} \gamma Q\right)^{-1} y
\end{array}
$$

which is equivalent to $K(Q)$ description in eq. (9) for right coprime factors.

According to the block diagram of structure 4 (fig. 4), $K(Q)$ yields:

$$
\begin{array}{r}
u=\left(K_{0}+\gamma Q^{\prime \prime} \tilde{M}_{0}\right)\left(I+\gamma Q^{\prime \prime} \tilde{N}_{0}\right)^{-1} y \\
u=\left(\tilde{V}_{0}^{-1} \tilde{U}_{0}+\gamma Q^{\prime \prime} \tilde{M}_{0}\right)\left(I+\gamma Q^{\prime \prime} \tilde{N}_{0}\right)^{-1} y \\
u=\left(\tilde{V}_{0}^{-1} \tilde{U}_{0}+\gamma Q \tilde{V}_{0}^{-1} \tilde{M}_{0}\right)\left(I+\gamma Q^{\prime \prime} \tilde{N}_{0}\right)^{-1} y \\
u=\left(\tilde{U}_{0}+\gamma Q \tilde{M}_{0}\right)\left(\tilde{V}_{0}+\gamma Q \tilde{N}_{0}\right)^{-1} y
\end{array}
$$

which is equivalent to $K(Q)$ description in eq. (9) for left coprime factors.

Stable controller reconfiguration property is ensured even if different structures are employed. Thus, CL poles during the transition keeps being the combination of the CL poles of $\left[G, K_{0}\right]$ and $\left[G, K_{1}\right]$.

\section{B. Structures 5 and 6}

Another critical point in the implementation of structures 1 and 2 is the inversion of coprime factors $V_{0}$ and $\tilde{V}_{0}$. As a solution, two new structures related to loop transfer recovery were proposed in [10] [13]. Structures so-called 5 and 6 do not present matrix inversion. Their block diagrams are depicted in fig. 5 and 6 . Notice that unlike structures 3 and 4 the calculation of the YK parameter $Q$ remains as in eq. (10), and the initial controller $K_{0}$ should be disconnected for structure implementation. Besides, the resulting controller order is the same that structures 1 and $2: 7\left(n+m_{0}\right)+3(n+$ $\left.m_{1}\right)$.

Again stable controller reconfiguration property is checked with eq. (9).

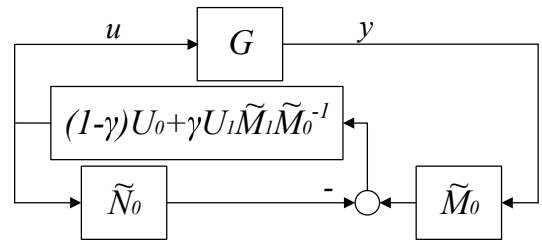

Fig. 7. Structure 7.

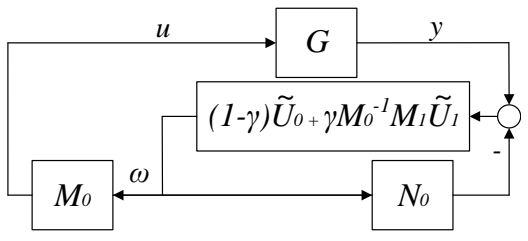

Fig. 8. Structure 8

According to the block diagram of structure 5 (fig. 5), $K(Q)$ yields:

$$
\begin{array}{r}
u=\left(U_{0}+M_{0} \gamma Q\right)\left(\tilde{M}_{0} y-\tilde{N}_{0} u\right)= \\
u=\left(I+\left(U_{0}+M_{0} \gamma Q\right) \tilde{N}_{0}\right)^{-1}\left(U_{0}+M_{0} \gamma Q\right) \tilde{M}_{0} y
\end{array}
$$

where according to Bézout's identity $M_{0} \tilde{N}_{0}=\tilde{M}_{0} N_{0}$ and $(I+$ $\left.U_{0} \tilde{N}_{0}\right)=\tilde{M}_{0} V_{0}$, yielding:

$$
\begin{array}{r}
u=\left(\tilde{M}_{0} V_{0}+\tilde{M}_{0} N_{0} \gamma Q\right)^{-1}\left(U_{0}+M_{0} \gamma Q\right) \tilde{M}_{0} y \\
u=\left(V_{0}+N_{0} \gamma Q\right)^{-1}\left(U_{0}+M_{0} \gamma Q\right) y
\end{array}
$$

which is equivalent to $K(Q)$ description in eq. (9) for right coprime factors.

According to the block diagram of structure 6 (fig. 6), $K(Q)$ yields:

$$
\begin{array}{r}
u=M_{0} \omega \\
u=M_{0}\left(\tilde{U}_{0}+\gamma Q \tilde{M}_{0}\right)\left(I+\left(\tilde{U}_{0}+\gamma Q \tilde{M}_{0}\right) N_{0}\right)^{-1} y \\
u=M_{0}\left(\tilde{U}_{0}+\gamma Q \tilde{M}_{0}\right)\left(\tilde{V}_{0} M_{0}+\gamma Q \tilde{N}_{0} M_{0}\right)^{-1} y \\
u=\left(\tilde{U}_{0}+\gamma Q \tilde{M}_{0}\right)\left(\tilde{V}_{0}+\gamma Q \tilde{N}_{0}\right)^{-1} u
\end{array}
$$

which is equivalent to $K(Q)$ description in eq. (9) for right coprime factors.

\section{Structures 7 and 8}

Finally, the work in [13] deals with the reduction of the implementation complexity by eliminating the YK parameter $Q$. Notice that this parameter is derived from eq. (10), which depends on six coprime factors. Structures 7 and 8 make the YK controller reconfiguration independent of $Q$. Control structures for right and left coprime factorizations are shown in figures 7 and 8 respectively. The equivalent complexity order results in $4\left(n+m_{0}\right)+2\left(n+m_{1}\right)$ states. Again structure implementation requires the initial controller to be disconnected. Notice how for the special case where $X_{1}=I$, the complexity order gets even lower.

Stable controller reconfiguration property is still preserved even if $\mathrm{Q}$ is no longer in the structure. Mathematical proof is shown below: 
According to the block diagram of structure 7 (fig. 7), $K(Q)$ yields:

$$
\begin{array}{r}
u=\left((1-\gamma) U_{0}+\gamma U_{1} \tilde{M}_{1} \tilde{M}_{0}{ }^{-1}\right)\left(\tilde{M}_{0} y-\tilde{N}_{0} u\right) \\
u=\left((1-\gamma) U_{0}+\gamma U_{1} \tilde{M}_{1}\left(-G U_{0}+V_{0}\right)\right)\left(\tilde{M}_{0} y-\tilde{N}_{0} u\right) \\
u=\left((1-\gamma) U_{0}+\gamma\left(-U_{1} \tilde{N}_{1} U_{0}+U_{1} \tilde{M}_{1} V_{0}\right)\right)\left(\tilde{M}_{0} y-\tilde{N}_{0} u\right) \\
u=\left((1-\gamma) U_{0}+\gamma\left(\left(I-M_{1} \tilde{V}_{1}\right) U_{1}+M_{1} \tilde{U}_{1} V_{0}\right)\right)\left(\tilde{M}_{0} y-\tilde{N}_{0} u\right) \\
u=\left(U_{0}+\gamma M_{1}\left(\tilde{U}_{1} V_{0}-\tilde{V}_{1} U_{0}\right)\right)\left(\tilde{M}_{0} y-\tilde{N}_{0} u\right) \\
u=\left(U_{0}+M_{0} \gamma Q\right)\left(\tilde{M}_{0} y-\tilde{N}_{0} u\right) \\
u=\left(I+\left(U_{0}+M_{0} \gamma Q\right) \tilde{N}_{0}\right)^{-1}\left(U_{0}+M_{0} \gamma Q\right) \tilde{M}_{0} y
\end{array}
$$

where according to the Bézout's identity $M_{0} \tilde{N}_{0}=\tilde{M}_{0} N_{0}$ and $\left(I+U_{0} \tilde{N}_{0}\right)=\tilde{M}_{0} V_{0}$, yielding:

$$
\begin{array}{r}
u=\left(\tilde{M}_{0} V_{0}+\tilde{M}_{0} N_{0} \gamma Q\right)^{-1}\left(U_{0}+M_{0} \gamma Q\right) \tilde{M}_{0} y \\
u=\left(V_{0}+N_{0} \gamma Q\right)^{-1}\left(U_{0}+M_{0} \gamma Q\right) y
\end{array}
$$

which is equivalent to $K(Q)$ description in eq. (9) for right coprime factors.

According to the block diagram of structure 8 (fig. 8), $K(Q)$ yields:

$$
\begin{array}{r}
u=M_{0} \omega \\
u=M_{0}\left((1-\gamma) \tilde{U}_{0}+\gamma M_{0}^{-1} M_{1} \tilde{U}_{1}\right) \\
\left(1+\left((1-\gamma) \tilde{U}_{0}+\gamma M_{0}^{-1} M_{1} \tilde{U}_{1}\right) N_{0}\right)^{-1} y \\
u=M_{0}\left(\tilde{U}_{0}+\gamma Q \tilde{M}_{0}\right)\left(1+\left(\tilde{U}_{0}+\gamma Q \tilde{M}_{0}\right) N_{0}\right)^{-1} y \\
u=M_{0}\left(\tilde{U}_{0}+\gamma Q \tilde{M}_{0}\right)\left(\tilde{V}_{0} M_{0}+\gamma Q \tilde{N}_{0} M_{0}\right)^{-1} y \\
u=\left(\tilde{U}_{0}+\gamma Q \tilde{M}_{0}\right)\left(\tilde{V}_{0}+\gamma Q \tilde{N}_{0}\right)^{-1} y
\end{array}
$$

which is equivalent to $K(Q)$ description in eq. (9) for left coprime factors.

On the other hand, it has been assumed that there is no variation in the system represented by $G$; when doing controller switching in a system with variations the dual YK formulation needs also to be employed. All the plants stabilized by a given controller are represented by $G(S)$, where $S$ is the dual YK parameter [17]. In that case, CL stability involves both $Q$ and $S:(I-Q S)^{-1} \in \mathbb{R} H_{\infty}$ [5]. Thus, control structures 7 and 8 cannot be used when system variations are present, as $Q$ is no longer in the structure.

\section{TRANSIENT BEHAVIOR}

By exploiting the YK parameterization it is possible to change controllers without losing stability, no matter what of the described control structures are used. As already mentioned, when doing controller transition $\gamma$ plays the key role as switching signal between $K_{0}$ and $K_{1}$. The rate of change of the switching signal $\gamma$ could be any, without affecting the CL stability of the system [6]. This section studies transient responses when using the different YK control structures, determining if the use of some structure improves the transient behavior when doing controller reconfiguration.

Notice that the rate of change of $\gamma$ could be any, but a numerical example with the fastest $\gamma$ rate is given such that transient differences are more remarkable.
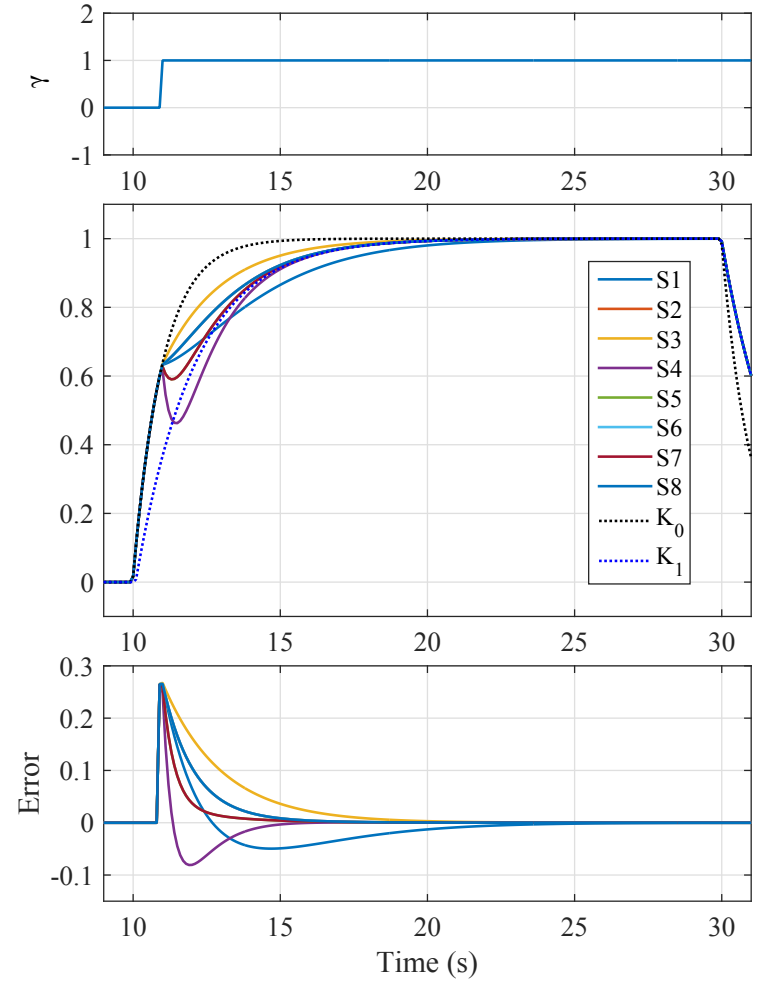

Fig. 9. Comparison of transient behavior between different YK control structures. Step from 0 to 1 .

Consider the following state space representation of a system:

$$
G(s)=\left[\begin{array}{c|c}
A & B \\
\hline C & D
\end{array}\right]=\left[\begin{array}{c|c}
-2.5 & 2.0 \\
\hline 1.25 & 0.0
\end{array}\right]
$$

A controller $K_{0}$ was designed to make the system follow a step reference:

$$
K_{0}(s)=\left[\begin{array}{c|c}
A_{0}^{c} & B_{0}^{c} \\
\hline C_{0}^{c} & D_{0}^{c}
\end{array}\right]=\left[\begin{array}{c|c}
0.0 & 1.0 \\
\hline-1.0 & -0.4
\end{array}\right]
$$

such that the following stable CL pole remains:

$$
\text { poles }_{C L\left(G, K_{0}\right)}=[-1]
$$

Later, the controller is replaced by a slower one:

$$
K_{1}(s)=\left[\begin{array}{c|c}
A_{1}^{c} & B_{1}^{c} \\
\hline C_{1}^{c} & D_{1}^{c}
\end{array}\right]=\left[\begin{array}{c|c}
0.0 & 0.5 \\
\hline-1.0 & -0.2
\end{array}\right]
$$

which CL pole is closer to the origin:

$$
\text { poles }_{C L\left(G, K_{1}\right)}=[-0.5]
$$

Responses of both controllers are shown as black and blue dot-lines in the middle graph of fig. 9.

Once $G, K_{0}$ and $K_{1}$ are defined, double coprime factors $N_{0}, M_{0}, N_{1}, M_{1}, \tilde{N}_{0}, \tilde{M}_{0}, \tilde{N}_{1}, \tilde{M}_{1}, U_{0}, V_{0}, U_{1}, V_{1}, \tilde{U}_{0}, \tilde{V}_{0}, \tilde{U}_{1}$, $\tilde{V}_{1}$ are obtained through Theorem 1 . These factors are used for the calculation of $Q, Q^{\prime}$ and $Q^{\prime \prime}$, so all the YK control structures can be implemented and compared in terms of transient performance.

Figure 9 depicts the transient behavior of each of the structures. The top graph represents how $\gamma$ is modified to 
TABLE I

SUMMARY TABLE.

\begin{tabular}{lccccccc}
\hline & $K_{0}$ disconnection & Complexity & Inversability & YK parameter & System variation $(\mathrm{Q}, \mathrm{S})$ & Time & Oscillations \\
\hline \hline Structure 1 & Yes & $7\left(n+m_{0}\right)+3\left(n+m_{1}\right)$ & Yes & Q & Yes & $12 s$ & Yes \\
\hline Structure 2 & Yes & $7\left(n+m_{0}\right)+3\left(n+m_{1}\right)$ & Yes & Q & Yes & $4 s$ & No \\
\hline Structure 3 & No & $5\left(n+m_{0}\right)+3\left(n+m_{1}\right)+m_{0}$ & No & Q' & Yes & $9 s$ & No \\
\hline Structure 4 & No & $5\left(n+m_{0}\right)+3\left(n+m_{1}\right)+m_{0}$ & No & Q" & Yes & $4 s$ & Yes \\
\hline Structure 5 & Yes & $7\left(n+m_{0}\right)+3\left(n+m_{1}\right)$ & No & Q & Yes & $2 s$ & Yes \\
\hline Structure 6 & Yes & $7\left(n+m_{0}\right)+3\left(n+m_{1}\right)$ & No & Q & Yes & $4 s$ & No \\
\hline Structure 7 & Yes & $4\left(n+m_{0}\right)+2\left(n+m_{1}\right)$ & Yes & - & No & $2 s$ & Yes \\
\hline Structure 8 & Yes & $4\left(n+m_{0}\right)+2\left(n+m_{1}\right)$ & Yes & - & No & $4 s$ & No \\
\hline
\end{tabular}

carry out the switching from $K_{0}$ to $K_{1}$. System responses when doing the transition are shown for the structures 1 to 8 at the middle graph. The bottom graph plots the error to $K_{1}$ response once the $\mathrm{YK}$ structure is activated. In all cases, the initial controller $K_{0}$ (black dot-line) is working until $\gamma$ becomes 1 at $11 s$. Then, the transient to reach $K_{1}$ response (blue dot-line) is different for each of the structures: Structures 5 and 7 presents the same response, the time to reach the desired behavior is the fastest $2 s$, but with an oscillation in the response; structures 2, 6 and 8 takes twice the time but without overshoot; Structure 3 is even slower, about $9 \mathrm{~s}$, but consequently transition is smoother; finally, structure 1 and 4 present oscillations over the desired response, with times reaching $12 s$ and $4 s$ respectively.

When doing controller reconfiguration, one looks for a transition without overshoot and as fast as possible between $K_{0}$ and $K_{1}$ responses. This is the case of structures 2, 6 and 8.

Table I gathers benefits and drawbacks of each structure seen in previous sections, as well as transition behavior characterized by settling time and the presence of oscillations.

\section{CONCLUSIONS}

This paper has explored the different YK control structures for stable controller reconfiguration.

The YK mathematical basis is explained and applied to derive the standard YK structures for controller switching. Once drawbacks of these structures are presented, different modifications are proposed to deal with problems such order complexity, plant disconnection or matrix inversability. Stability property is still preserved despite modifications in the structure, which means that CL poles during transition are the same even if different structures are used.

Even if stability and CL poles are maintained during transition, transient behavior of each structure is investigated. Transient responses results different depending on the used YK controller structure. Structures 1, 4, 5 and 7 exhibit an oscillating response to be avoided. The rest present acceptable behaviors, but one or the other should be chosen depending on the desired time to reach the final response and the controller complexity. Structures 2, 6 and 8 are the fastest ones without oscillation.

Finally, the different properties mentioned throughout the paper are summarized in table I. This table can serve as a guide to choose the appropiate structure, according to the expected system performance.

\section{ACKNOWLEDGMENT}

This work has been founded by French National Research Agency via the VALET project (ANR-15-CE22-0013).

\section{REFERENCES}

[1] K. Ogata, "Modern control engineering,(1997)," ISBN: 0-13-2273071, pp. 299-231, 2013.

[2] H. Niemann, J. Stoustrup, and R. B. Abrahamsen, "Switching between multivariable controllers," Optimal control applications and methods, vol. 25, no. 2, pp. 51-66, 2004.

[3] V. Kucera, Discrete linear control: the polynomial equation approach. John Wiley \& Sons, Inc., 1980.

[4] J. B. Moore, K. Glover, and A. Telford, "All stabilizing controllers as frequency-shaped state estimate feedback," IEEE Transactions on Automatic Control, vol. 35, no. 2, pp. 203-208, 1990.

[5] H. Niemann and J. Stoustrup, "An architecture for implementation of multivariable controllers," in American Control Conference, 1999. Proceedings of the 1999, vol. 6. IEEE, 1999, pp. 4029-4033.

[6] J. P. Hespanha and A. S. Morse, "Switching between stabilizing controllers," Automatica, vol. 38, no. 11, pp. 1905-1917, 2002.

[7] T.-T. Tay, I. Mareels, and J. B. Moore, High performance control. Springer Science \& Business Media, 2012.

[8] H. Niemann and N. K. Poulsen, "Fault tolerant control for uncertain systems with parametric faults," IFAC Proceedings Volumes, vol. 39, no. 13 , pp. 480-485, 2006.

[9] J. Stoustrup, "Plug \& play control: Control technology towards new challenges," in European Journal of Control, vol. 15, no. 3-4, 2009, pp. 311-330.

[10] H. H. Ntemann, P. Søgaard-Andersen, and J. Stoustrup, "Loop transfer recovery for general observer architectures," International Journal of Control, vol. 53, no. 5, pp. 1177-1203, 1991.

[11] K. Trangbaek, J. Stoustrup, and J. Bendtsen, "Stable controller reconfiguration through terminal connections," IFAC Proceedings Volumes, vol. 41 , no. 2, pp. 331-335, 2008.

[12] K. Trangbaek and J. Bendtsen, "Stable controller reconfiguration through terminal connectionsa practical example," in Control and Automation, 2009. ICCA 2009. IEEE International Conference on. IEEE, 2009, pp. 2037-2042.

[13] H. Niemann and N. K. Poulsen, "Controller architectures for switching," in American Control Conference, 2009. ACC'09. IEEE, 2009, pp. $1098-1103$.

[14] K. Zhou, J. Doyle, and K. Glover., Robust and Optimal Control, P. Hall, Ed., 1996.

[15] C. Nett, C. Jacobson, and M. Balas, "A connection between state-space and doubly coprime fractional representations," IEEE Transactions on Automatic Control, vol. 29, no. 9, pp. 831-832, 1984.

[16] J. F. Pommaret and A. Quadrat, "Generalized bezout identity," in Aplicable Algebra in Engineering, Communication and Computing, vol. 9, no. 2, 1998, pp. 91-116.

[17] J. Bendtsen and K. Trangbaek, "Closed-loop identification for control of linear parameter varying systems," Asian Journal of Control, vol. 16, no. 1, pp. 40-49, 2014. 